\title{
Consistent Integration of Models Based on Views of Meta Models
}

\author{
Hartmut Ehrig, Karsten Ehrig, Claudia Ermel and Ulrike Prange \\ Institut für Softwaretechnik und Theoretische Informatik, \\ Technische Universität Berlin, Germany
}

\begin{abstract}
The complexity of large system models in software engineering nowadays is mastered by using different views. View-based modelling aims at creating small, partial models, each one of them describing some aspect of the system. Existing formal techniques supporting view-based visual modelling are based on typed attributed graphs, where views are related by typed attributed graph morphisms. Such morphisms up to now require a meta model given by a fixed type graph, as well as a fixed data signature and domain. This is in general not adequate for view-oriented modeling where only parts of the complete meta model are known and necessary when modelling a partial view of the system.

The aim of this paper is to extend the framework of typed attributed graph morphisms to generalized typed attributed graph morphisms, short GAG-morphisms, which involve changes of the type graph, data signature, and domain. This allows the modeller to formulate type hierarchies and views of visual languages defined by GAG-morphisms between type graphs, short GATG-morphisms. In this paper, we study the interaction and integration of views, and the restriction of views along type hierarchies. In the main result, we present suitable conditions for the integration and decomposition of consistent view models (Theorem 4.1) and extend these conditions to view models defined over meta models with constraints (Theorem 5.1). As a running example, we use a visual domain-specific modelling language to model coarse-grained IT components and their connectors in decentralized IT infrastructures. Using constraints, we formulate connection properties as invariants.
\end{abstract}

Keywords: meta-modelling, views of visual languages, generalized typed attributed graph morphisms, view interaction, view integration

\section{Introduction}

In recent years, the complexity of large system models in software engineering is mastered by using different views or viewpoints. View-based modeling rather aims at creating small, partial models, each one of them describing some aspect of the system instead of building complex monolithic specifications. Visual techniques nowadays form an important part of the overall software development methodology. Usually, visual notations

Correspondence and offprint requests to: Claudia Ermel, Technische Universität Berlin, Sekr. FR6-1, Franklinstr. 28-29, D 10587 Berlin, Germany. e-mail: claudia.ermel@tu-berlin.de 
like the UML [OMG07], Petri nets or other kinds of graphs are used in order to specify static or dynamic system aspects. Hence, the syntax definition of visual modeling languages is an important basis for the implementation of tools supporting visual modeling (e.g. visual editor generation) and for model-based system verification.

Two main approaches to visual language (VL) definition can be distinguished: grammar-based approaches or meta-modeling. Using graph grammars and graph transformation [EEPT06], multidimensional representations are described by graphs. Graph rules are used to manipulate the graph representation of a language element. Meta-modeling (see e.g. [MOF06]) is also graph-based, but uses constraints instead of a grammar to define a visual language. The advantage of meta-modeling is that UML users, who probably have basic UML knowledge, do not need to learn a new external notation to be able to deal with syntax definitions. Graph grammars are more constructive, i.e. closer to the implementation, and provide a formal basis for visualizing, validating and verifying system properties.

For the application of graph transformation techniques to VL modeling, typed attributed graph transformation systems and grammars [EEPT06] have proven to be an adequate formalism. A VL is modeled by a type graph capturing the definition of the underlying visual alphabet, i.e. the symbols and relations which are available. Sentences or models of the VL are given by graphs typed over (i.e. conforming to) the type graph. Such a VL type graph corresponds closely to a meta model. In order to restrict the set of valid visual models, a syntax graph grammar may be defined, consisting of a set of language-generating graph transformation rules, typed over the abstract syntax part of the VL type graph.

In this paper, we extend the graph transformation framework in order to allow an adequate specification of different views and their relations. In the literature, approaches already exist to model views as morphisms between typed attributed graphs [EEHT97]. Up to now such morphisms require a fixed type graph, as well as a fixed data signature and domain. This is in general not adequate for view-oriented modeling where only parts of the complete type graph and signature are known and necessary when modeling a partial view of the system. Hence, in this paper we develop the notion of generalized attributed graph morphisms (GAG-morphisms) which allows the modeler to change the type graph, data signature and domain. GAGmorphisms are the basis for more flexible, view-oriented modeling since views are independent of each other, now also with respect to the data type definition.

For view-oriented modeling, mechanisms are needed to integrate different views to a full system model. In order to integrate two or more views, their intended correspondences have to be specified. Here, typed graphs and the underlying categorical constructions support an integration concept which goes much further than an integration merely based on the use of common names. In this paper, we define type hierarchies and views based on GAG-morphisms, and study the interaction and integration of views, as well as the restriction of views along type hierarchies, the notion of view consistency, and the integration and decomposition of models based on consistent views.

As a running example we use a visual domain-specific modeling language to model coarse-grained IT components and their connectors in decentralized IT infrastructures. An infrastructure model has to provide the basis to handle structural security issues, like firewall placements, of such distributed IT components. In order to provide support to model, build, administrate, monitor and control such a local IT landscape, we present a formal, visual domain-specific language family based on attributed type graph hierarchies and views. A simplified visual language for this purpose using typed graphs without attributes was first introduced in $\left[\mathrm{BBE}^{+} 07\right]$, serving as a basis to transform domain-specific IT infrastructure models to a Reo coordination model [Arb04] for further analysis.

The paper is structured as follows: Section 2 defines the category GAGraphs of typed attributed graphs and GAG-morphisms, and introduces the sample VL for IT infrastructures. On this basis, views are defined in Section 3, and the view relations interaction and integration are given by categorical constructions. Moreover, the interplay of type hierarchies of VLs and views is considered. Section 4 studies models of visual languages and models of views (view-models) and states as main result conditions for the consistency, integration and decomposition of view-models. Type hierarchies and views with constraints are studied in Section 5, an extension which is not included in our conference version [EEEP08]. In Section 6, related work is presented and compared to our approach. We conclude and discuss future work in Section 7. 


\section{Visual Language Definition by Typed Attributed Graphs}

We use the meta-model approach in combination with typed attributed graphs to define visual languages. A meta-model is given by an attributed type graph $A T G$ together with structural constraints, and the corresponding visual language $V L$ is given by all attributed graphs typed over $A T G$ which satisfy the constraints. In the following, we introduce the necessary definitions for typed attributed graphs.

The definition of attributed graphs is based on E-graphs, which give a structure for graphs with data elements.

An E-graph $G=\left(V_{G}, V_{D}, E_{G}, E_{N A}, E_{E A},\left(\text { source }_{j}, \text { target }_{j}\right)_{j \in\{G, N A, E A\}}\right)$ has two different kinds of nodes, namely graph nodes $V_{G}$ and data nodes $V_{D}$, and different kinds of edges, namely graph edges $E_{G}$ and, for the attribution, node attribute edges $E_{N A}$ and edge attribute edges $E_{E A}$, with corresponding source and target functions according to the signature on the right.

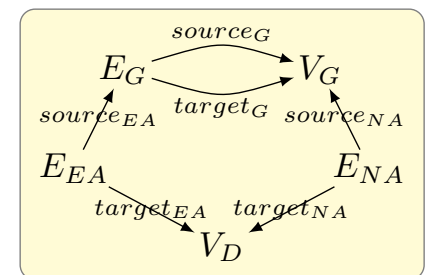

As presented in [EEPT06], attributed graphs are defined as E-graphs combined with a DSIG-algebra, i.e. an algebra over a data signature $D S I G$. In this signature, we distinguish a set of attribute value sorts. The corresponding carrier sets in the $D S I G$-algebra can be used for attribution. In addition to attributed graph morphisms in [EEPT06], generalized attributed graph morphisms are mappings of attributed graphs with possibly different data signatures.

Definition 2.1 (Attributed graph and generalized attributed graph morphism). An attributed graph $A G=(G, D S I G, D)$ consists of

- an E-graph $G=\left(V_{G}, V_{D}, E_{G}, E_{N A}, E_{E A},\left(\text { source }_{j} \text { target }_{j}\right)_{j \in\{G, N A, E A\}}\right)$,

- a data signature $D S I G=\left(S, S_{D}, O P\right)$ with attribute value sorts $S_{D} \subseteq S$, and

- a $D S I G$-algebra $D$ such that $\underset{s \in S_{D}}{ } D_{s}=V_{D}$.

Given attributed graphs $A G^{i}=\left(G^{i}, D S I G^{i}, D^{i}\right)$ for $i=1,2$, a generalized attributed graph morphism (GAG-morphism) $f=\left(f_{G}, f_{S}, f_{D}\right): A G^{1} \rightarrow A G^{2}$ is given by

- an E-graph morphism $f_{G}: G^{1} \rightarrow G^{2}$,

- a signature morphism $f_{S}: D S I G^{1} \rightarrow D S I G^{2}$, and

- a generalized homomorphism $f_{D}: D^{1} \rightarrow D^{2}$, which is a $D S I G^{1}$-morphism $f_{D}: D^{1} \rightarrow V_{f_{S}}\left(D^{2}\right)$ with $f_{D}=\left(f_{D, s_{1}}: D_{s_{1}}^{1} \rightarrow D_{f_{S}\left(s_{1}\right)}^{2}\right)_{s_{1} \in S^{1}}$

with the following compatibility property: $f_{S}\left(S_{D}^{1}\right) \subseteq S_{D}^{2}$ and the diagram on the right commutes for all $s_{1} \in S_{D}^{1}$, where the vertical (curling) arrows are inclusions.

A GAG-morphism $f=\left(f_{G}, f_{S}, f_{D}\right)$ is called

- injective, if $f_{G}, f_{S}, f_{D}$ are injective,

- signature preserving, if $f_{S}$ is isomorphic,

- persistent, if $f_{D}$ is isomorphic.

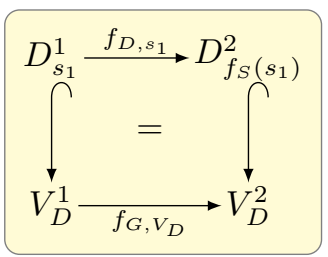

Attributed graphs with generalized attributed graph morphisms form the category GAGraphs.

Note that AG-morphisms in [EEPT06] correspond to signature preserving GAG-morphisms. For the typing, we use a distinguished attributed type graph ATG. According to [EEPT06], attributed type graphs and typed attributed graphs are now defined using GAG-morphisms presented above.

Definition 2.2 (Typed attributed graph and typed attributed graph morphism).

An attributed type graph $A T G=\left(T G, D S I G, Z_{D S I G}\right)$ is an attributed graph where $Z_{D S I G}$ is the final $D S I G$-algebra, i.e. $Z_{D S I G, s}=\{s\}$ for all $s \in S$, and $V_{D}=\dot{\cup}_{s \in S_{D}} Z_{D S I G, s}=S_{D}$. 
Given an attributed type graph $A T G$, a typed attributed graph $T A G=(A G, t)$ (over $A T G$ ) is given by an attributed graph $A G$ and a GAG-morphism $t: A G \rightarrow A T G$.

Given an attributed type graph $A T G$ and typed attributed graphs $T A G^{i}=\left(A G^{i}, t: A G^{i} \rightarrow A T G\right)$ over $A T G$ for $i=1,2$, a typed attributed graph morphism $f: T A G^{1} \rightarrow T A G^{2}$ is given by a GAG-morphism $f: A G^{1} \rightarrow A G^{2}$ such that $t_{2} \circ f=t^{1}$.

Given an attributed type graph $A T G$, typed attributed graphs over $A T G$ and typed attributed graph morphisms form the category GAGraphs $\mathbf{A T G}$.

As a special case of GAG-morphisms we obtain generalized attributed type graph morphisms based on attributed type graphs.

Definition 2.3 (Generalized attributed type graph morphism).

Given attributed type graphs $A T G^{i}=\left(T G^{i}, D S I G^{i}, Z_{D S I G^{i}}\right)$ for $i=1,2$, a generalized attributed type graph morphism (GATG-morphism) $f=\left(f_{G}, f_{S}, f_{D}\right): A T G^{1} \rightarrow A T G^{2}$ is given by

- an E-graph morphism $f_{G}: T G^{1} \rightarrow T G^{2}$,

- a signature morphism $f_{S}: D S I G^{1} \rightarrow D S I G^{2}$, and

- a generalized homomorphism $f_{D}: Z_{D S I G^{1}} \rightarrow Z_{D S I G^{2}}$, which is uniquely determined by $f_{D, s_{1}}\left(s_{1}\right)=$ $f_{S}\left(s_{1}\right)$ for all $s_{1} \in S^{1}$.

A GATG-morphism $f$ is also a GAG-morphism since the compatibility property is automatically satisfied because $f_{G, V_{D}}\left(s_{1}\right)=f_{S}\left(s_{1}\right)$ for all $s_{1} \in S_{D}^{1}$ and $f_{D}, f_{G, V_{D}}$ are uniquely determined by $f_{S}$. Moreover, if $f$ is a GATG-morphism then $f$ is persistent.

Now we are able to define visual languages. In this section, we consider only visual languages over attributed type graphs, without any constraints. We deal with visual language based on meta models with constraints in Section 5.

Definition 2.4 (Visual language).

Given an attributed type graph $A T G$, the visual language $V L$ of $A T G$ consists of all typed attributed graphs $(A G, t: A G \rightarrow A T G)$ typed over $A T G$, i.e. $V L$ is the object class of the category GAGraphs ATG $_{\text {. }}$

Example 2.1 (VL for network infrastructures).

Fig. 1 shows at the top the attributed type graph $A T G_{D S L}$ which represents a meta-meta model (or schema) for domain-specific languages for IT infrastructures. The $D S L$ schema defines that all its instances (domainspecific languages) consist of node types for components, connections and interfaces. In the center of Fig. 1, the attributed type graph $A T G_{N e t w o r k}$ defines a simple modeling language for network infrastructures which has component types for personal computers (PC), application servers (AS), and databases (DB). Interfaces are refined into HTTP-client and HTTP-server ports, as well as database client and server ports. Connections may be secure (i.e. with firewall) or insecure, which is modeled by the new boolean attribute secure.

There is a generalized attributed type graph morphism $h$ from $A T G_{N e t w o r k}$ to $A T G_{D S L}$, indicated by equal numbering of mapped nodes. Note that in order to be able to define the signature morphism $f_{S}$ and the $D S I G$-morphism $f_{D}$ for any GAG-morphisms $f: A T G_{1} \rightarrow A T G_{2}$ between different type graphs, we assume that each node type in $A T G_{2}$ has at least one sort "**, and one attribute attr: $*$, where all sorts and attributes from $A T G_{1}$ can be mapped to which are not already defined in $A T G_{2}$. Thus we can have new attributes, sorts and methods at the more detailed type level $A T G_{1}$ which need not be defined already in $A T G_{2}$. For our sample GAG-morphism $h$ in Fig. 1, this is the case for the new attribute secure : Bool of the type Connection in $A T G_{N e t w o r k}$. The new sort Bool is mapped by the signature morphism to the sort "*", and the attribute secure is mapped by the $D S I G$-morphism to the constant attr.

At the bottom of Fig. 1, a sample computer network is depicted as graph $G_{N e t w o r k}$ which is an element of the visual Network language since $G_{\text {Network }}$ is typed over $A T G_{\text {Network }}:\left(G_{\text {Network }}, t: G \rightarrow A T G_{\text {Network }}\right) \in$ $V L_{N e t w o r k}$. Obviously, all graphs $G$ in $V L_{N e t w o r k}$ are also in $V L_{D S L}$, since every $\left(G, t: G \rightarrow A T G_{N e t w o r k}\right)$ is also typed over $A T G_{D S L}$ by the composition of typing morphisms: $\left(G, h \circ t: G \rightarrow A T G_{D S L}\right) \in V L_{D S L}$.

\section{Type Hierarchies and Views of Visual Languages and Meta Models}

In this section, we study type hierarchies and views of visual languages based on morphisms in GAGraphs, which allow to change not only the graph structure but also the data signature and data type. Note that in 


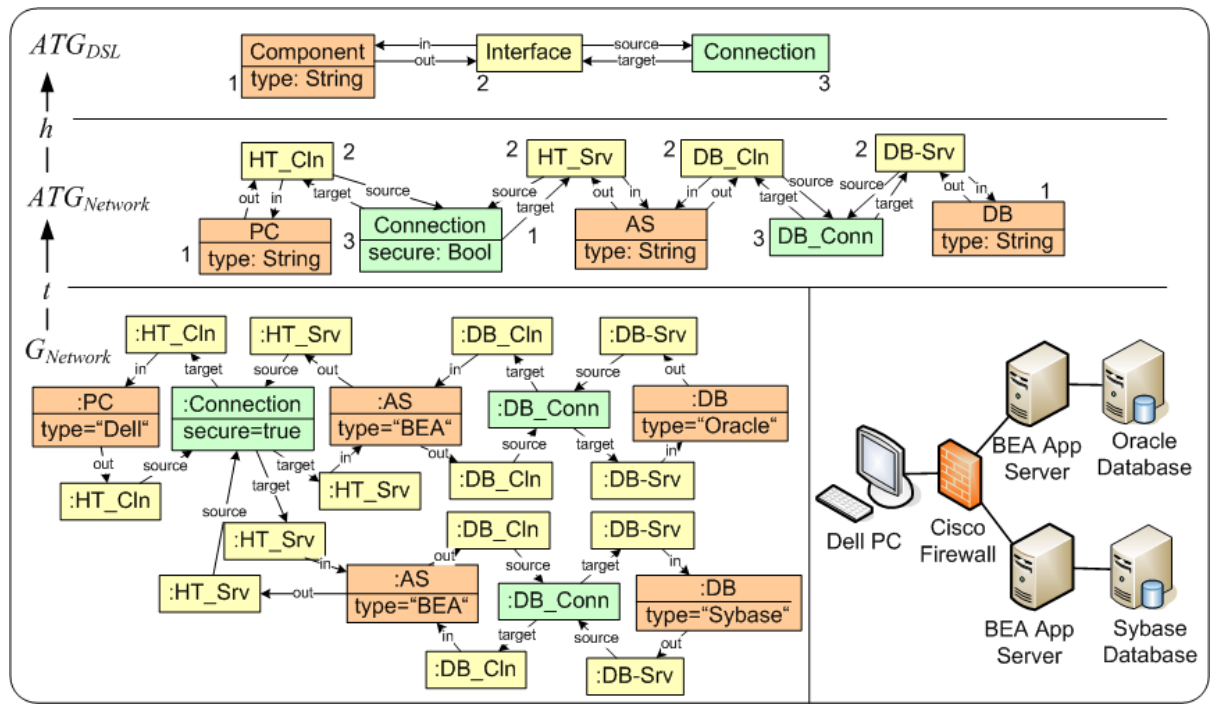

Fig. 1. Example 2.1: Domain-Specific Languages for IT Infrastructures

this section, we only consider the attributed type graphs and their relations, but not yet models over them. This is done in the next section.

A restriction of a visual language to a specific subpart of the language is called a view.

Definition 3.1 (View). A view of a visual language $V L$ over an attributed type graph $A T G$ - also called view of the meta model $A T G$ - is given by an injective GATG-morphism $v_{1}: A T G_{1} \rightarrow A T G$.

For the interaction and integration of views we need the categorical constructions of pullbacks and pushouts in GAGraphs. Proofs for the pushout and pullback construction lemmas are given in [EEEP09]. Pullbacks are a kind of generalized intersection of objects over a common object.

Fact 3.1 (Pullback construction in GAGraphs). Given GAG-morphisms $f: A G^{2} \rightarrow A G^{3}$ and $g$ : $A G^{1} \rightarrow A G^{3}$ then the pullback in GAGraphs is constructed componentwise in the $G$-, $S$ - and $D$ components. Moreover, pullbacks preserve injective, signature preserving, and persistent morphisms.

Proof. See [EEEP09].

Pushouts generalize the gluing of objects, i.e. a pushout emerges from the gluing of two objects along a common sub-object using the amalgamation of data types in the sense of [EM85].

Fact 3.2 (Pushouts in GAGraphs over persistent morphisms). Given persistent morphisms $f^{\prime}$ : $A G^{0} \rightarrow A G^{1}$ and $g^{\prime}: A G^{0} \rightarrow A G^{2}$ in GAGraphs then the pushout (1) in GAGraphs is constructed componentwise in the $G$ - and $S$-components, with attribute value sorts $S_{D}^{3}=g_{s}\left(S_{D}^{1}\right) \cup f_{S}\left(S_{D}^{2}\right)$, and in the $D$-component by amalgamation as $D_{3}=D^{1}+{ }_{D^{0}} D^{2}$. Moreover, pushouts preserve injective, signature preserving, and persistent morphisms.

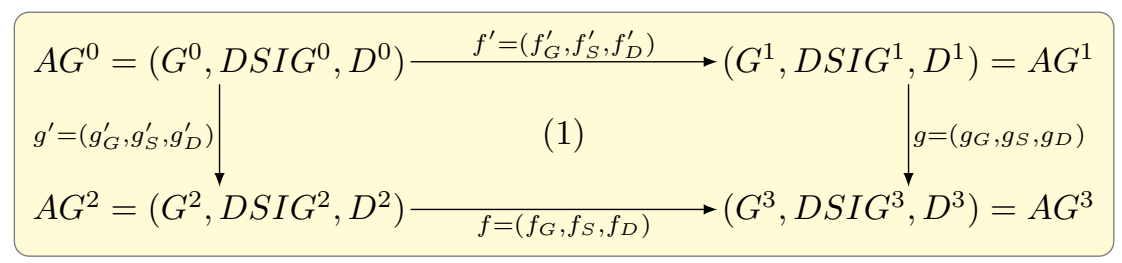

Proof. See [EEEP09].

Remark. Moreover, we show in [EEEP09] that the category (GAGraphs, $\mathcal{M}$ ) with the class $\mathcal{M}$ of all injective, persistent, and signature preserving morphisms and also the corresponding typed variant 
(GAGraphs ATG $\stackrel{M}{\longrightarrow}$ ) are adhesive HLR categories. This allows us to apply main parts of the theory for typed attributed graph transformations developed on the basis of the categories (AGraphs, $\mathcal{M}$ ) and $\left(\right.$ AGraphs $\left._{\text {ATG }}, \mathcal{M}\right)$, respectively, also to the generalized case. The main difference is that graphs in GAGraphs $_{\text {ATG }}$ allow for the typing $t: A G \rightarrow A T G$ a change of the data type signature.

We are now able to define the interaction and integration of views based on the concepts of pullbacks and pushouts. Roughly speaking, the interaction is the intersection, and the integration is the union of views.

Definition 3.2 (Interaction and integration of views). Given views $\left(A T G_{1}, v_{1}\right)$ and $\left(A T G_{2}, v_{2}\right)$ over $A T G$ the interaction $\left(A T G_{0}, i_{1}, i_{2}\right)$ is given by the following pullback (1) in GAGraphs, where $\left(A T G_{0}, v_{0}\right)$ with $v_{0}=v_{1} \circ i_{1}=v_{2} \circ i_{2}$ is a view over $A T G$ and also called subview of $\left(A T G_{1}, v_{1}\right)$ and $\left(A T G_{2}, v_{2}\right)$.
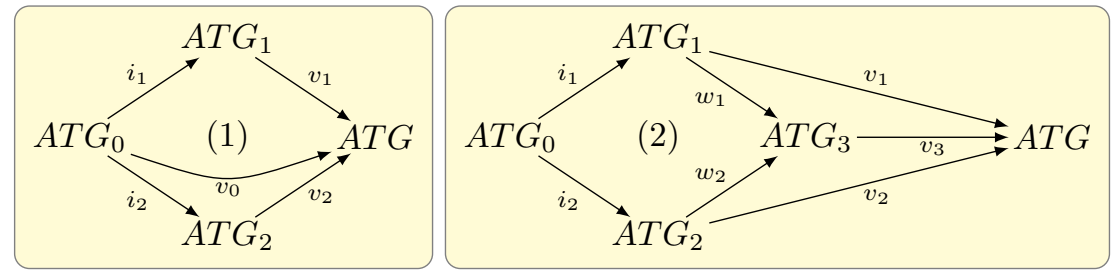

The integration of views $\left(A T G_{1}, v_{1}\right)$ and $\left(A T G_{2}, v_{2}\right)$ with interaction $\left(A T G_{0}, i_{1}, i_{2}\right)$ is given by the above pushout (2) in GAGraphs. Due to the universal pushout property there is a unique injective GATGmorphism $v_{3}: A T G_{3} \rightarrow A T G$ such that $\left(A T G_{3}, v_{3}\right)$ is a view over $A T G$.

$A T G$ is covered by views $\left(A T G_{i}, v_{i}\right)$ with $i=1,2$ if $v_{1}$ and $v_{2}$ are jointly surjective.

There is a close relationship between covering by views and view integration.

Fact 3.3 (Integration of views). If $A T G$ is covered by views $\left(A T G_{i}, v_{i}\right)$ for $i=1,2$ then the integration $A T G_{3}$ is equal to $A T G$ up to isomorphism.

Proof. According to Def. 3.2, there is a unique morphism $v_{3}$ with $v_{3} \circ w_{1}=v_{1}$ and $v_{3} \circ w_{2}=v_{2}$. This morphism is injective in the $G$ - and $S$-components due to general properties of graph and signature morphisms, and $v_{3}$ is injective in the $D$-component as a general property of GATG-morphisms. Surjectivity of $v_{3}$ follows from joint surjectivity of $v_{1}$ and $v_{2}$.

Example 3.1 (Interaction and integration of views on IT networks). Fig. 2 shows two views $\left(A T G_{\text {Components }}, v_{1}\right)$ and $\left(A T G_{\text {Connections }}, v_{2}\right)$ of the visual language over $A T G_{D S L}$ (see Fig. 1). The type graph $A T G_{\text {Components }}$ consists of a node type for Computer linked to a node type for Port, whereas the type graph $A T G_{\text {Connections }}$ contains a node type Channel which is linked to a node type ChEnd. The view embedding $v_{1}$ maps Computer to Component and Port to Interface, and $v_{2}$ maps Channel to Connection and ChEnd to Interface. Edges are mapped accordingly. The interaction $\left(A T G_{\text {interaction }}, i_{1}, i_{2}\right)$ is constructed as pullback (1) in GAGraphs which is the intersection of $v_{1}$ and $v_{2}$ with suitable renaming. Given the interaction, the integration of the views $\left(A T G_{\text {Components }}, v_{1}\right)$ and $A T G_{\text {Connections }}, v_{2}$ over $\left(A T G_{\text {Interaction }}, i_{1}, i_{2}\right)$ can be constructed as pushout $(2)$ in GAGraphs, resulting in the type graph $\left(A T G_{\text {Integration }}\right)$. According to Fact $3.3,\left(A T G_{\text {Integration }}\right)$ is isomorphic to $A T G_{D S L}$, since $A T G_{D S L}$ is covered by $\left(A T G_{\text {Components }}, v_{1}\right)$ and $\left(A T G_{\text {Connections }}, v_{2}\right)$.

In order to support stepwise language development, visual languages can be structured hierarchically: one attributed type graph $A T G$ may specify the abstract concepts a set of visual languages $V L_{i}$ have in common, and different type graphs $A T G_{i}$ for these visual languages refine the types in $A T G$ by specifying multiple concrete subtypes for them. The type hierarchy relation is formalized by GATG-morphisms $h_{i}$ from $A T G_{i}$ to $A T G$. The morphism $h: A T G_{\text {Network }} \rightarrow A T G_{D S L}$ depicted in Fig. 1 is such a type hierarchy morphism. The next step is to define the restriction of views along type hierarchies by pullbacks.

Definition 3.3 (Type hierarchy and restriction of views). A type hierarchy of visual languages $V L$ and $V L^{\prime}$ given by attributed type graphs $A T G$ and $A T G^{\prime}$, respectively, is a GATG-morphism $h: A T G^{\prime} \rightarrow$ $A T G$. 


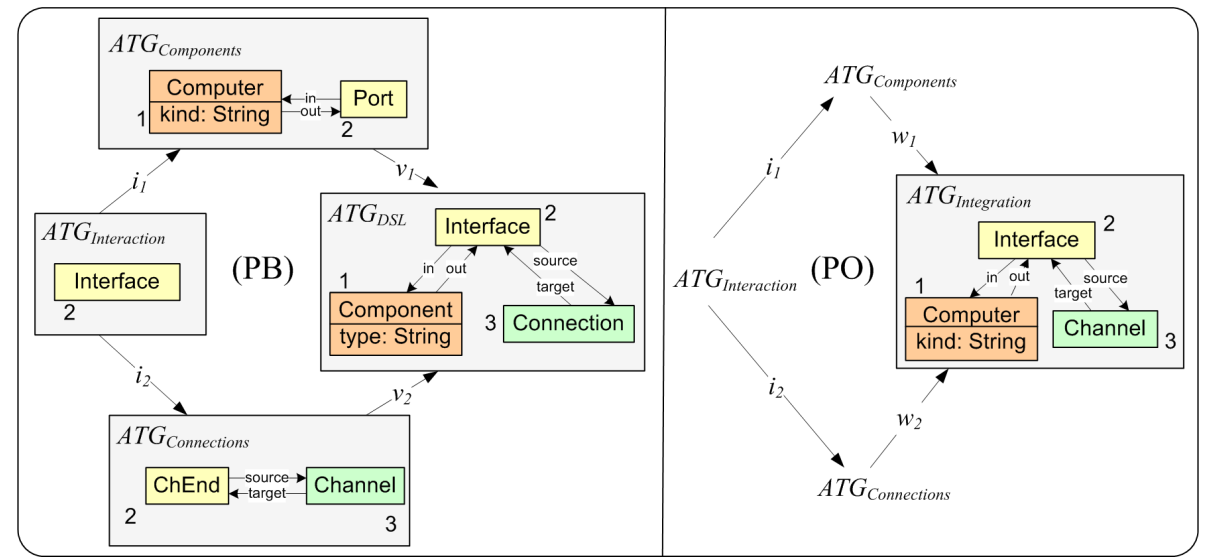

Fig. 2. Example 3.1: Interaction and Integration of two Views on $A T G_{D S L}$

Given a type hierarchy morphism $h: A T G^{\prime} \rightarrow A T G$ and a view $\left(A T G_{1}, v_{1}\right)$ over $A T G$ then the restriction $\left(A T G_{1}^{\prime}, v_{1}^{\prime}\right)$ of this view along $h$ is defined by the pullback (1) in GAGraphs.

The restriction $\left(A T G_{1}^{\prime}, v_{1}^{\prime}\right)$ is a view over $A T G^{\prime}$ because pullbacks preserve injectivity.

Fact 3.4 (Hierarchy and covering views). Given a hierarchy morphism $h: A T G^{\prime} \rightarrow A T G$ and views $\left(A T G_{i}, v_{i}\right)$ for $i=1,2$ covering $A T G$, then the restrictions $\left(A T G_{i}^{\prime}, v_{i}^{\prime}\right)$ along $h$ are covering $A T G^{\prime}$.

Proof. In the diagram to the right, $v_{1}$ and $v_{2}$ being jointly surjective implies that also $v_{1}^{\prime}$ and $v_{2}^{\prime}$ are jointly surjective because (1) and (2) are componentwise pullbacks.
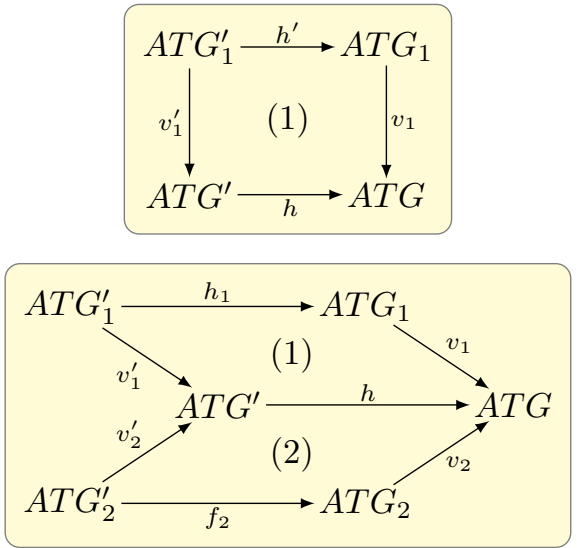

Example 3.2 (Hierarchy and covering views). The morphism $h: A T G_{\text {Network }} \rightarrow A T G_{D S L}$ in Fig. 1 is a type hierarchy morphism. Moreover, we have two views $\left(A T G_{\text {Components }}, v_{1}\right)$ and $\left(A T G_{\text {Connections }}, v_{2}\right)$ on $A T G_{D S L}$, shown in Fig. 2, which are covering $A T G_{D S L}$. Fig. 3 shows the restrictions $v_{1}^{\prime}$ and $v_{2}^{\prime}$ of the views along the hierarchy morphism $h$ which are covering $A T G_{\text {Network }}$ due to Fact 3.4.

\section{Models and View-Models of Visual Languages}

In this section, we study models of visual languages and models of views of visual languages, called viewmodels, and we present our main result on the integration and decomposition of models.

Definition 4.1 (Model). Given a meta-model of a visual language $V L$ by an attributed type graph $A T G$, then a model of $V L$ is a typed attributed graph $A G$, typed over $A T G$ with a GAG-morphism $t: A G \rightarrow A T G$.

The model $(A G, t)$ is called signature-conform if $t$ is signature-preserving.

Similar to the restriction of views at the type level we now define the restriction of models at the model level.

Definition 4.2 (Restriction). Given a view $f: A T G_{1} \rightarrow$ $A T G$, i.e. an injective GATG-morphism, and an $A T G$-model $(A G, t)$ then the restriction $\left(A G_{1}, t_{1}\right)$ of $(A G, t)$ to the view $\left(A T G_{1}, f\right)$ is defined by the pullback $(1)$, written $f^{<}(A G, t)=$ $\left(A G_{1}, t_{1}\right)$.

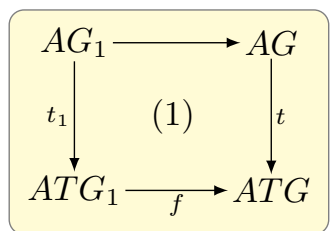




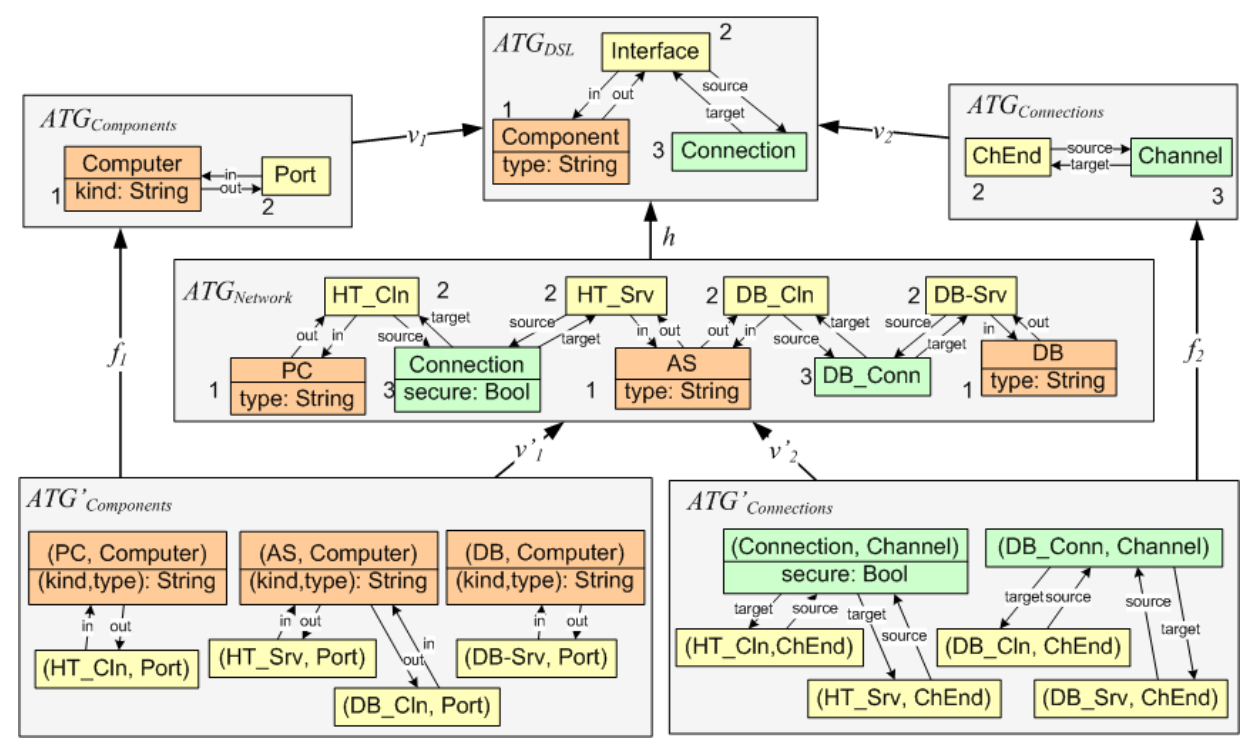

Fig. 3. Example 3.2: Restriction of two Views along Hierarchy Morphism $h$

The construction $f^{<}(A G, t)$ is called backward typing and can be extended to a functor $f<(A G, t)$ : GAGraphs $_{\mathbf{A T G}} \rightarrow \mathbf{G A G r a p h s}_{\mathbf{A T G}_{1}}$, as opposed to the extension of view models defined by forward typing $f^{>}\left(A G_{1}, t_{1}\right)=\left(A G_{1}, f \circ t_{1}\right)$.

In order to state the main result on integration and decomposition of models, we have to define the notions of consistency and integration for models. Roughly, models $A G_{1}$ and $A G_{2}$ of type $A T G_{1}$ and $A T G_{2}$, respectively, are consistent if they agree on the interaction type $A T G_{0}$. In this case, there is an integrated model $A G$ such that the restrictions of $A G$ to $A T G_{1}$ and to $A T G_{2}$ are equal to the given models $A G_{1}$ and $A G_{2}$, respectively.

Definition 4.3 (Consistency and integration). Given views $\left(A T G_{i}, v_{i}\right)$ for $i \quad=\quad 1,2$ of $A T G$ with interaction $\left(A T G_{0}, i_{1}, i_{2}\right)$ defined by the pullback in the bottom face of the following cube, then the models $\left(A G_{i}, t_{i}\right)$ of the views $\left(A T G_{i}, v_{i}\right)$ are called consistent if there is a model $\left(A G_{0}, t_{0}\right)$ of $A T G_{0}$ such that the back faces are pullbacks, i.e. $i_{1}^{<}\left(A G_{1}, t_{1}\right)=\left(A G_{0}, t_{0}\right)=i_{2}^{<}\left(A G_{2}, t_{2}\right)$.

A model $(A G, t)$ of $A T G$ is called integration (or amalgamation) of consistent $\left(A G_{1}, t_{1}\right)$ and $\left(A G_{2}, t_{2}\right)$ via $\left(A G_{0}, t_{0}\right)$ if the front faces of the above cube are pullbacks, i.e. $v_{1}^{<}(A G, t)=\left(A G_{1}, t_{1}\right)$ and $v_{2}^{<}(A G, t)=\left(A G_{2}, t_{2}\right)$, and the top face commutes.

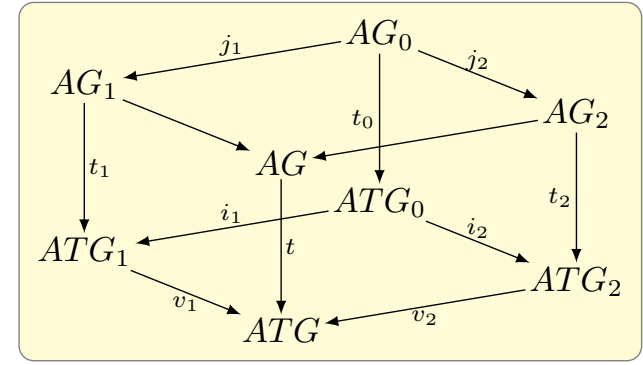

Example 4.1 (Inconsistent models). Consider the view models $A G_{1}$ and $A G_{2}$ in Fig. 4. These models are inconsistent since the squares (1) and (2) are pullbacks corresponding to the back squares of the cube in Def. 4.3, but the resulting pullback objects $A G_{0}$ and $A G_{0}^{\prime}$ are different (and non-isomorphic), so we have $i_{1}^{<}\left(A G_{1}, t_{1}\right)=\left(A G_{0}, t_{0}\right) \neq i_{2}^{<}\left(A G_{2}, t_{2}\right)=\left(A G_{0}^{\prime}, t_{0}^{\prime}\right)$. In this case, there is no integration $(A G, t)$ s.t. $v_{1}^{<}(A G, t)=\left(A G_{1}, t_{1}\right)$ and $v_{2}^{<}(A G, t)=\left(A G_{2}, t_{2}\right)$.

Theorem 4.1 (Integration and decomposition of models). Let $A T G$ be covered by the views $\left(A T G_{i}, v_{i}\right)$ for $i=1,2$.

Integration. If $\left(A G_{i}, t_{i}\right)$ are consistent models of $\left(A T G_{i}, v_{i}\right)$ via $\left(A G_{0}, t_{0}\right)$ then there is up to isomorphism a unique integration $(A G, t)$ of $\left(A G_{i}, t_{i}\right)$ via $\left(A G_{0}, t_{0}\right)$.

Decomposition. Vice versa, each model $(A G, t)$ of $A T G$ can be decomposed uniquely up to isomorphism 


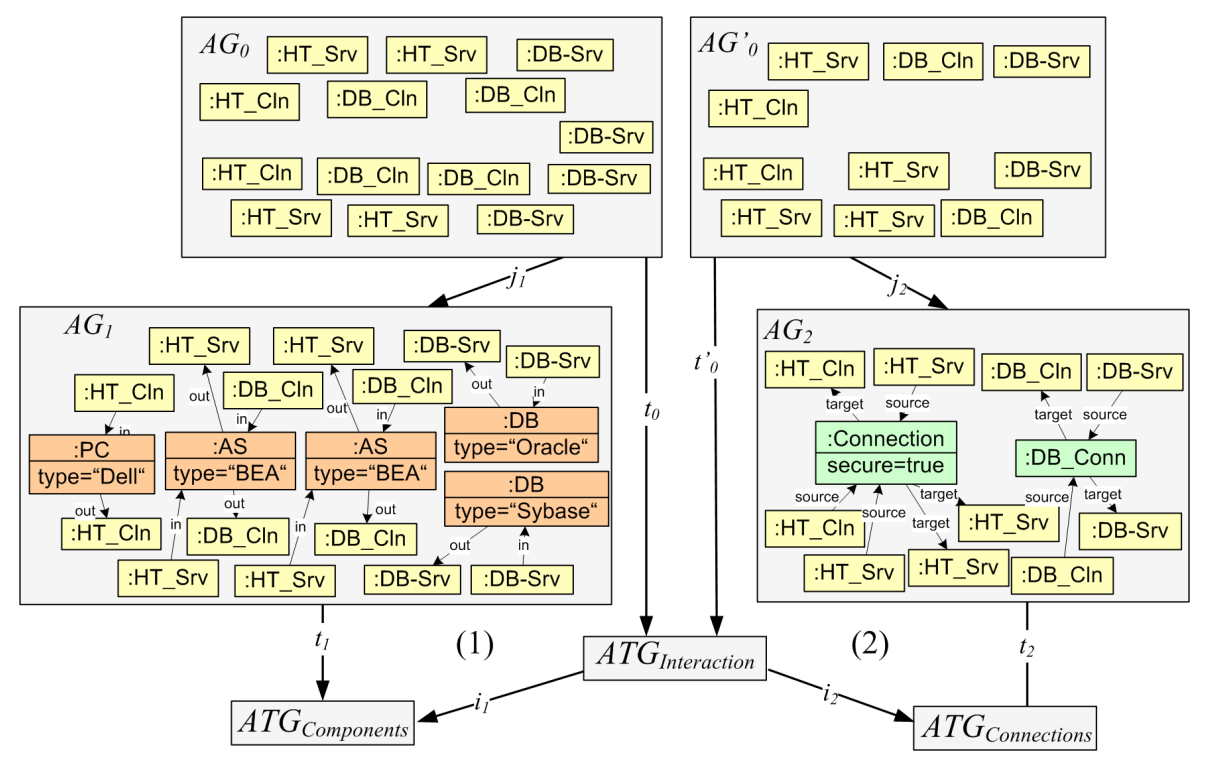

Fig. 4. Example 4.1: Inconsistent View Models

into view-models $\left(A G_{i}, t_{i}\right)$ with $i=1,2$ such that $(A G, t)$ is the integration of $\left(A G_{1}, t_{1}\right)$ and $\left(A G_{2}, t_{2}\right)$ via $\left(A G_{0}, t_{0}\right)$.

Bijective Correspondence. Integration and decomposition are inverse to each other up to isomorphism.

Proof.

Integration. Since $A T G$ is covered by $\left(A T G_{i}, v_{i}\right)$ for $i=1,2$ it is also the integration of these views by Fact 3.3. This means that the bottom pullback in the cube in Def. 4.3 is already a pushout in GAGraphs with injective and persistent morphisms. Now assume that $\left(A G_{i}, t_{i}\right)$ with $i=1,2$ are consistent models. This means that the back faces of the cube in Def. 4.3 are pullbacks with injective and persistent $j_{1}$ and $j_{2}$. This allows to construct $A G$ in the top face as pushout in GAGraphs leading to a unique $t$ such that the front faces commute. According to a suitable van Kampen property (see [EEEP09]), the front faces are pullbacks such that $(A G, t)$ is the integration of $\left(A G_{i}, t_{i}\right)$ for $i=1,2$ via $\left(A G_{0}, t_{0}\right)$. In order to show the uniqueness let also $\left(A G^{\prime}, t^{\prime}: A G^{\prime} \rightarrow A T G\right)$ be an integration of $\left(A G_{i}, t_{i}\right)$ for $i=1,2$ via $\left(A G_{0}, t_{0}\right)$. Then the front faces are pullbacks with $\left(A G^{\prime}, t^{\prime}\right)$ and the top face commutes. Now the van Kampen property in the opposite direction implies that the top face is a pushout in GAGraphs. This implies that $(A G, t)$ and $\left(A G^{\prime}, t^{\prime}\right)$ are equal up to isomorphism.

Decomposition. Vice versa, given a model $(A G, t)$ of $A T G$ we construct the front and one of the back faces as pullbacks such that the remaining back face also becomes a pullback and the top face commutes. This shows that $\left(A G_{1}, t_{1}\right)$ and $\left(A G_{2}, t_{2}\right)$ are consistent w.r.t $\left(A G_{0}, t_{0}\right)$, and, similar to the previous step, $(A G, t)$ is the integration of both via $\left(A G_{0}, t_{0}\right)$. The decomposition is unique up to isomorphism because the pullbacks in the front faces are unique up to isomorphism.

Bijective Correspondence. Uniqueness of integration and decomposition as shown above implies that both constructions are inverse to each other up to isomorphism.

Example 4.2 (Integration and decomposition of models). The graph $G_{\text {Network }}$ from Fig. 1 is a model, typed over $A T G_{\text {Network }}$. From the two views $A T G_{\text {Components }}^{\prime}$ and $A T G_{\text {Connections }}^{\prime}$ given in Fig. 3 we can construct two consistent view models $G_{\text {Components }}$ and $G_{\text {Connections }}$ in Fig. 5 according to the Decomposition in Thm. 4.1 such that $G_{\text {Network }}$ is the integration of $G_{\text {Components }}$ and $G_{\text {Connections }}$ via $G_{\text {interaction }}$. Vice versa, starting with consistent models $G_{\text {Components }}$ and $G_{\text {Connections }}$, via $G_{\text {interaction }}$ we obtain $G_{\text {Network }}$ as the integration. 


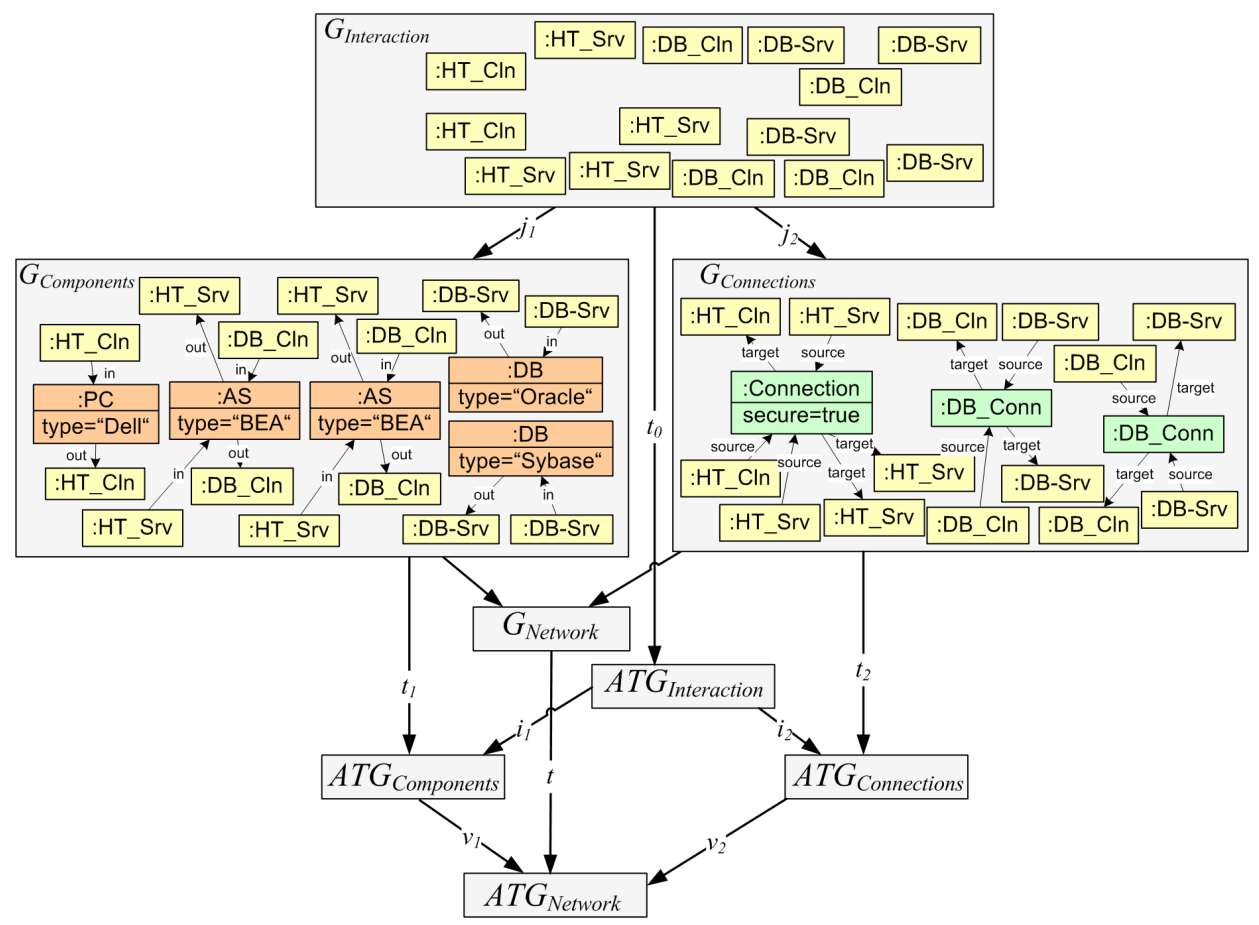

Fig. 5. Example 4.2: Integration and Decomposition of View Models

\section{Type Hierarchies and Views with Constraints}

In this section, we extend the definition of a visual language $V L$ given by an attributed type graph $A T G$ by a set of graph constraints $P C$ which pose further restrictions on the set of valid visual models in a natural, visual way. A visual language definition given by a type graph and a set of graph constraints corresponds closely to a meta model according to the MOF approach [MOF06], together with a set of OCL constraints [OCL03].

\section{Definition 5.1 (Graph constraint).}

Let $A T G$ be an attributed type graph. A constraint $c=\left(\left(P, t_{P}\right) \stackrel{a}{\longrightarrow}\left(C, t_{C}\right)\right)$ is given by typed attributed graphs $\left(P, t_{P}\right)$ and $\left(C, t_{C}\right)$ typed over $A T G$, where we omit the typing morphisms if they are not necessary, i.e. write $c=(P \stackrel{a}{\longrightarrow} C)$, and a typed attributed graph morphism $a: P \rightarrow C$. A model $G$ typed over $A T G$ fulfills a constraint $c=(P \stackrel{a}{\longrightarrow} C)$ if for all typed attributed graph morphisms $p: P \rightarrow G$ there exists an injective $q: C \rightarrow G$ such that $q \circ a=p$.

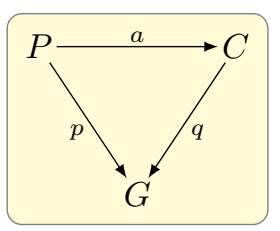

Definition 5.2 (Visual language with constraints). A visual language over a type graph $A T G$ and a set of constraints $P C$ is defined by $V L=\left\{G \in \mathbf{G A G r a p h s}_{\mathbf{A T G}} \mid G \models c \forall c \in P C\right\}$.

The following facts concern the satisfaction of constraints in view models which are extended or restricted to different type graphs:

Definition 5.3 (Forward translation of constraints). Given a GATG-morphism $f: A T G_{1} \rightarrow A T G_{2}$ and a constraint $c_{1}=\left(\left(P, t_{P}\right) \stackrel{a}{\longrightarrow}\left(C, t_{C}\right)\right)$ over $A T G_{1}$, the forward translated constraint $f^{>}\left(c_{1}\right)=c_{2}$ over $A T G_{2}$ is given by $c_{2}=\left(\left(P, f \circ t_{P}\right) \stackrel{a}{\longrightarrow}\left(C, f \circ t_{C}\right)\right)$. For a set $P C_{1}$ of constraints over $A T G_{1}$, we define $f^{>}\left(P C_{1}\right)=\left\{f^{>}\left(c_{1}\right) \mid c_{1} \in P C_{1}\right\}$.

Fact 5.1 states that a forward translated constraint is satisfied by an extended view model whenever the originally typed constraint is satisfied by the original view model, and vice versa. 
Fact 5.1. Given a view $\left(A T G_{1}, v_{1}\right)$ over $A T G_{2}$, a constraint $c_{1} \in P C_{1}$ typed over $A T G_{1}$, and a typed attributed graph $G_{1}$ typed over $A T G_{1}$, then we have:

$$
G_{1} \models c_{1} \Leftrightarrow v_{1}^{>}\left(G_{1}\right) \models v_{1}^{>}\left(c_{1}\right),
$$

where $v_{1}^{>}\left(G_{1}\right)$ and $v_{1}^{>}\left(c_{1}\right)$ are the corresponding forward translations over $A T G_{2}$.

Proof. For $c_{1}=\left(\left(P, t_{P}\right) \stackrel{a}{\longrightarrow}\left(C, t_{C}\right)\right)$ we have $v_{1}^{>}\left(c_{1}\right)=\left(\left(P, v_{1} \circ t_{P}\right) \stackrel{a}{\longrightarrow}\left(C, v_{1} \circ t_{C}\right)\right)$, and $v_{1}^{>}\left(G_{1}, t_{G_{1}}\right)=\left(G_{1}, v_{1} \circ t_{G_{1}}\right)$.

" $\Rightarrow$ " We have to show that for each injective $p: P \rightarrow G_{1}$ in GAGraphs $\mathbf{A T G}_{\mathbf{2}}$ there is an injective $q: C \rightarrow G_{1}$ in GAGraphs $_{\mathbf{A T G}_{\mathbf{2}}}$ with $q \circ a=p$. Given an injective $p: P \rightarrow G_{1}$ in GAGraphs ATG $_{2}$ we have $p: P \rightarrow G_{1}$ in GAGraphs with $v_{1} \circ t_{P}=v_{1} \circ t_{G_{1}} \circ p$. Since $v_{1}$ is injective it follows that $t_{P}=t_{G_{1}} \circ p$, i.e. $p$ is also an $\mathbf{G A G r a p h s} \mathbf{A T G}_{\mathbf{1}}$-morphism. Since $G_{1} \models c_{1}$ there exists an injective $q: C \rightarrow G_{1}$ with $q \circ a=p$ in GAGraphs $_{\mathbf{A T G}_{\mathbf{1}}}$, i.e. $t_{G_{1}} \circ q=t_{C}$. Hence $v_{1} \circ t_{G_{1}} \circ q=v_{1} \circ t_{C}$ and $q$ is the required $\mathbf{G A G r a p h s} \mathbf{A T G}_{\mathbf{2}}$-morphism.

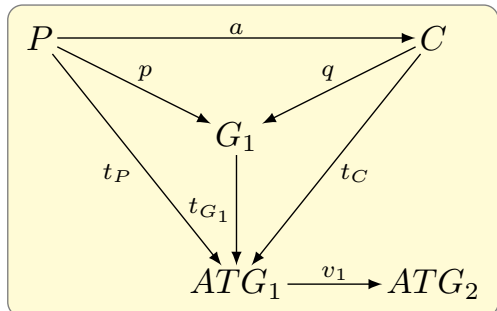

" $\Leftarrow$ " We have to show that for each injective $p: P \rightarrow G_{1}$ in GAGraphs $_{\mathbf{A T G}_{1}}$ there is an injective $q: C \rightarrow G_{1}$ in GAGraphs $\mathbf{A T G}_{\boldsymbol{1}}$ with $q \circ a=p$. Given an injective $p: P \rightarrow G_{1}$ in $\mathbf{G A G r a p h s}_{\mathbf{A T G}_{\mathbf{1}}}$ we have $p: P \rightarrow G_{1}$ in GAGraphs with $t_{P}=t_{G_{1}} \circ p$. With $v_{1} \circ t_{P}=v_{1} \circ t_{G_{1}} \circ p, p$ is also a GAGraphs $\mathbf{A T G}_{2}$-morphism. Since $v_{1}^{>}\left(G_{1}\right) \models v_{1}^{>}\left(c_{1}\right)$ there exists an injective $q: C \rightarrow G_{1}$ with $q \circ a=p$ in GAGraphs $\mathbf{A T G}_{2}$, i.e. $v_{1} \circ t_{G_{1}} \circ q=v_{1} \circ t_{C}$. Since $v_{1}$ is injective it follows that $t_{G_{1}} \circ q=t_{C}$, hence $q$ is the required GAGraphs $\mathbf{A T G}_{\mathbf{1}}$-morphism.

Example 5.1. Consider the constraint "An application server always has two HTTP-server ports", shown in Fig. 6 in the upper right corner as constraint $c=\left(\left(P, t_{P}\right) \stackrel{a}{\longrightarrow}\left(C, t_{C}\right)\right)$. This constraint is typed originally

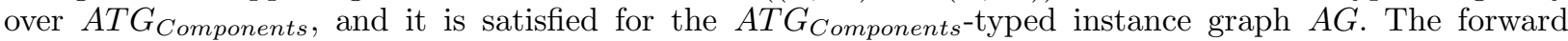
translation of constraint $c$ is given by the constraint $c^{\prime}=\left(\left(\left(P, v \circ t_{P}\right) \stackrel{a}{\longrightarrow}\left(C, v \circ t_{C}\right)\right)\right.$, typed over $A T G_{N e t w o r k}$. Obviously, constraint $c^{\prime}$ is satisfied for graph $A G$, which is also typed over $A T G_{N e t w o r k}$ by typing morphism $A G \stackrel{v \circ t}{\longrightarrow} A T G_{\text {Network }}$.

Fact 5.2 states that a forward translated constraint is satisfied by a model whenever the original constraint is satisfied by the corresponding restricted view model, and vice versa.

Fact 5.2. Given a view $\left(A T G_{1}, v_{1}\right)$ over $A T G_{2}$, a constraint $c_{1} \in P C_{1}$ typed over $A T G_{1}$, and a typed attributed graph $G_{2}$ typed over $A T G_{2}$, then we have:

$$
G_{2} \models v_{1}^{>}\left(c_{1}\right) \Leftrightarrow v_{1}^{<}\left(G_{2}\right) \models c_{1},
$$

where $v_{1}^{>}\left(c_{1}\right)$ is the forward translation of $c_{1}$ and $v_{1}^{<}\left(G_{2}\right)$ is the backward translation of $G_{2}$.

Proof. For $c_{1}=\left(\left(P, t_{P}\right) \stackrel{a}{\longrightarrow}\left(C, t_{C}\right)\right)$ we have $v_{1}^{>}\left(c_{1}\right)=\left(\left(P, v_{1} \circ t_{P}\right) \stackrel{a}{\longrightarrow}\left(C, v_{1} \circ t_{C}\right)\right)$, and $v_{1}^{<}\left(G_{2}, t_{G_{2}}\right)=\left(G_{1}, t_{G_{1}}\right)$ with pullback $(1)$.

" $\Rightarrow$ "We have to show that for each injective $p_{1}: P \rightarrow G_{1}$ in GAGraphs $\mathbf{A T G}_{\mathbf{1}}$ there is an injective $q_{1}: C \rightarrow G_{1}$ in GAGraphs ATG $_{1}$ with $q_{1} \circ a=p_{1}$. Given an injective $p_{1}: P \rightarrow G_{1}$ in $\mathbf{G A G r a p h s}_{\mathbf{A T G}_{1}}$, with $v_{1}$ being injective and (1) being a pullback also $g$ and hence $g \circ p_{1}$ are injective. Thus we have that $t_{G_{2}} \circ g \circ p_{1}=$ $v_{1} \circ t_{G_{1}} \circ p_{1}=v_{1} \circ t_{P}$ and since $G_{2} \models v_{1}^{>}\left(c_{1}\right)$ there exists an injective $q_{2}: C \rightarrow G_{2}$ with $q_{2} \circ a=g \circ p_{1}$ in GAGraphs $_{\mathbf{A T G}_{\mathbf{2}}}$, i.e. $t_{G_{2}} \circ q_{2}=v_{1} \circ t_{C}$. Now pullback (1) implies a unique $q_{1}: C \rightarrow G_{1}$ with $t_{G_{1}} \circ q_{1}=t_{C}$ and $g \circ q_{1}=q_{2}$. The latter implies that $q_{1}$ is injective by decomposition of monomorphisms. Hence $q_{1}$ is the

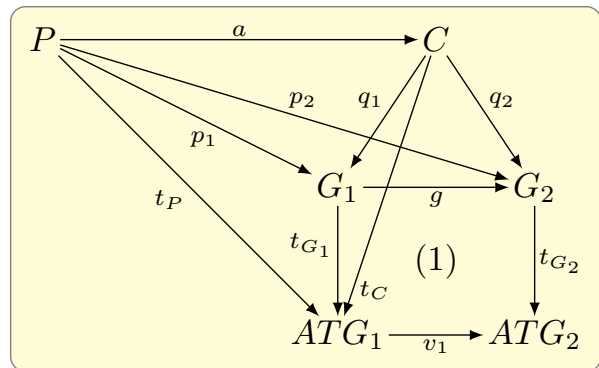
required $\mathbf{G A G r a p h s}_{\mathbf{A T G}_{\mathbf{1}}}$-morphism. 


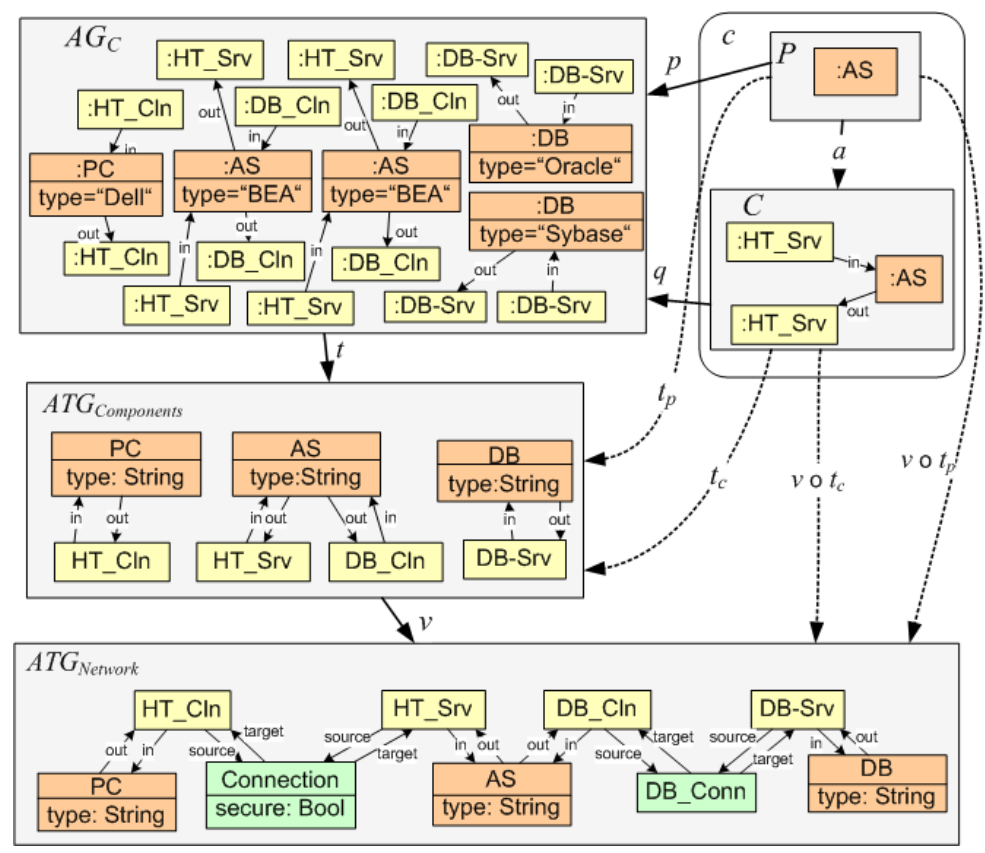

Fig. 6. Example 5.1: Forward translation of constraints

"६" We have to show that for each injective $p_{2}: P \rightarrow G_{2}$ in $\mathbf{G A G r a p h s}_{\mathbf{A T G}_{2}}$ there is an injective $q_{2}: C \rightarrow G_{2}$ in GAGraphs $\mathbf{A T G}_{\mathbf{2}}$ with $q_{2} \circ a=p_{2}$. Given an injective $p_{2}: P \rightarrow G_{2}$ in GAGraphs $\mathbf{A T G}_{\mathbf{2}}$ we have $t_{G_{2}} \circ p_{2}=v_{1} \circ t_{P}$. Pullback (1) implies a unique $p_{1}: P \rightarrow G_{1}$ with $t_{G_{1}} \circ p_{1}=t_{P}$ and $g \circ p_{1}=p_{2}$. The latter implies that $p_{1}$ is injective by decomposition of monomorphisms. Since $G_{1} \models c_{1}$ there exists an injective $q_{1}: C \rightarrow G_{1}$ with $q_{1} \circ a=p_{1}$ in GAGraphs $\mathbf{A T G}_{\mathbf{1}}$, i.e. $t_{G_{1}} \circ q_{1}=t_{C}$. It follows that $g \circ q_{1}$ is injective. Thus we have that $t_{G_{2}} \circ g \circ q_{1}=v_{1} \circ t_{G_{1}} \circ q_{1}=v_{1} \circ t_{C}$. Hence $q_{2}=g \circ q_{1}$ is the required GAGraphs $_{\mathbf{A T G}_{\mathbf{2}}}$-morphism with $q_{2} \circ a=g \circ q_{1} \circ a=g \circ p_{1}=p_{2}$.

Example 5.2. In Fig. 7, the constraint $c$ is originally typed over $A T G_{\text {Components. Its forward translation }}$ $c^{\prime}=\left(\left(\left(P, v \circ t_{P}\right) \stackrel{a}{\longrightarrow}\left(C, v \circ t_{C}\right)\right)\right.$ is typed over $A T G_{N e t w o r k}$, and it is satisfied for the $A T G_{N e t w o r k}$-typed instance model $A G_{\text {Network }}$. The view model of $A G_{\text {Network }}$ over the view $A T G_{\text {Components }} \stackrel{v}{\longrightarrow} A T G_{N e t w o r k}$ is obtained by constructing the pullback $(P B)$ and yields as pullback object the $A T G_{\text {Components }}$-typed model $A G_{C}$ which was shown explicitly in Fig. 6. Moreover, from Example 5.1 we know that the constraint $c$ is satisfied by $A G_{C}$.

Fact 5.3 considers the satisfaction of sets of constraints by extended and restricted view models. We find that constraint implication preserves visual language extensions and reflects visual language restrictions.

Fact 5.3. Given attributed type graphs $A T G_{1}$ and $A T G_{2}$, constraints $P C_{1}$ and $P C_{2}$ over $A T G_{1}$ and $A T G_{2}$ leading to visual languages $V L_{1}$ and $V L_{2}$, respectively, and a view $\left(A T G_{1}, v_{1}\right)$ over $A T G_{2}$, then we have the following results:

1. (VL extension) If $v_{1}^{>}\left(P C_{1}\right) \Rightarrow P C_{2}$ then $v_{1}^{>}\left(G_{1}\right) \in V L_{2}$ for all $G_{1} \in V L_{1}$, i.e. $v_{1}^{>}: V L_{1} \rightarrow V L_{2}$.

2. (VL restriction) If $P C_{2} \Rightarrow v_{1}^{>}\left(P C_{1}\right)$ then $v_{1}^{<}\left(G_{2}\right) \in V L_{1}$ for all $G_{2} \in V L_{2}$, i,e, $v_{1}^{<}: V L_{2} \rightarrow V L_{1}$.

Proof. 1. Given $G_{1} \in V L_{1}$ this means that $G_{1} \models P C_{1}$. Now Fact 5.1 implies that $v_{1}^{>}\left(G_{1}\right) \models v_{1}^{>}\left(P C_{1}\right)$ and if $v_{1}^{>}\left(P C_{1}\right) \Rightarrow P C_{2}$ also $v_{1}^{>}\left(G_{1}\right)=P C_{2}$, i.e. $v_{1}^{>}\left(G_{1}\right) \in V L_{2}$.

2. Given $G_{2} \in V L_{2}$ this means that $G_{2} \models P C_{2}$, and if $P C_{2} \Rightarrow v_{1}^{>}\left(P C_{1}\right)$ also $G_{2} \models v_{1}^{>}\left(P C_{1}\right)$. Now Fact 5.2 implies that $v_{1}^{<}\left(G_{2}\right) \models P C_{1}$, i.e. $v_{1}^{<}\left(G_{2}\right) \in V L_{1}$. 


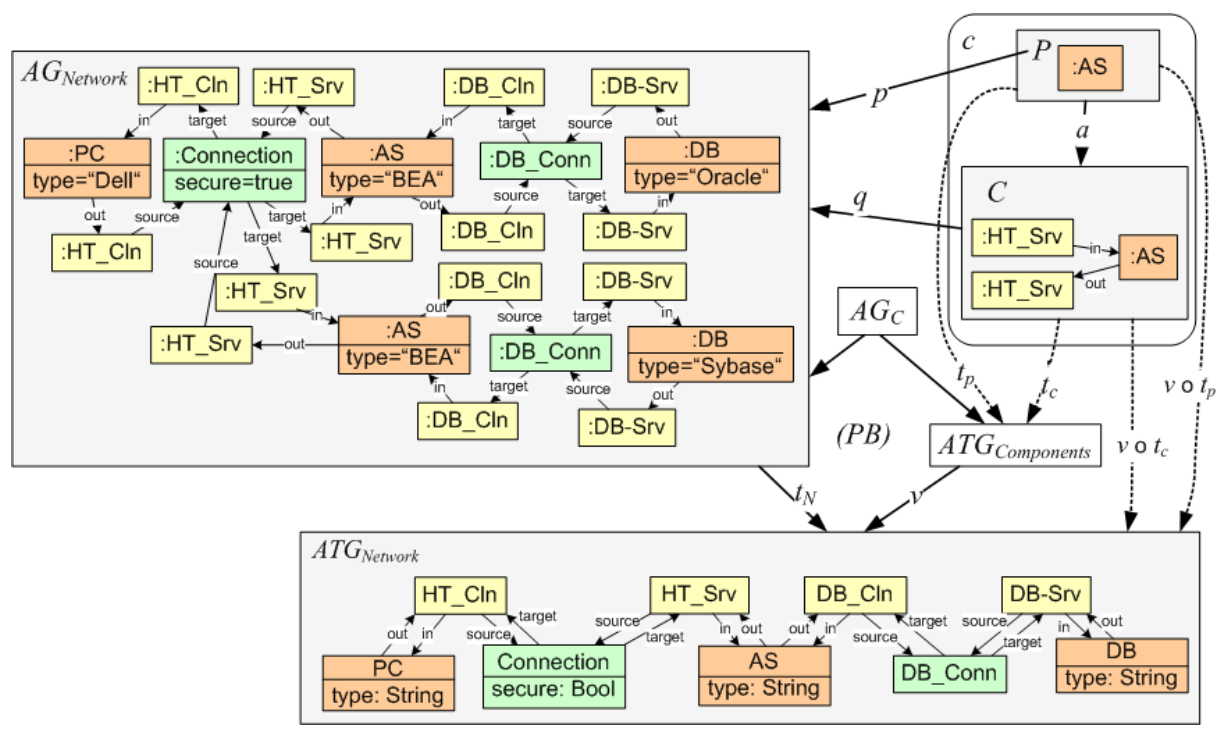

Fig. 7. Example 5.2: Backward translation of constraints

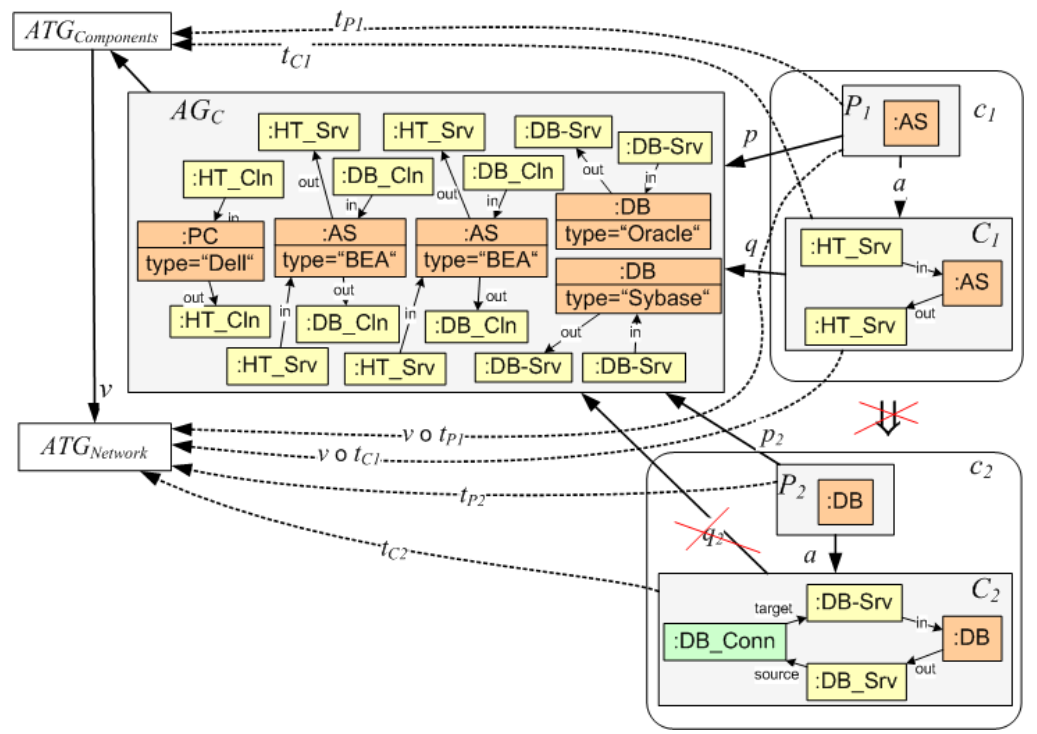

Fig. 8. Example 5.3: Constraint implication preserves forward-translated languages

\section{Example 5.3.}

1. Consider again the constraint "An application server always has two HTTP-server ports", shown in Fig. 8 in the upper right corner as constraint $c_{1}=\left(\left(P_{1}, t_{P 1}\right) \stackrel{a}{\longrightarrow}\left(C_{1}, t_{C 1}\right)\right)$. As shown in Example 5.1, the forward translation of constraint $c_{1}$, given by the constraint $c_{1}^{\prime}=\left(\left(\left(P, v \circ t_{P 1}\right) \stackrel{a}{\longrightarrow}\left(C, v \circ t_{C 1}\right)\right)\right.$, is typed over $A T G_{\text {Network }}$ and is satisfied for graph $A G_{C}$. Obviously, constraint $c_{1}^{\prime}$ does not imply constraint $c_{2}$ which requires that every database server is connected via two DB server interface nodes to a database connection. This constraint is not satisfied by the forward-translated model $A G_{C}$. Hence, model $A G_{C}$ does not belong to the visual language defined by the type graph $A T G_{N e t w o r k}$ and a set of constraints $P C_{2}$ with $c_{2} \in P C_{2}$.

2. In Fig. 9, the constraint $c_{2}$, typed over $A T G_{\text {Network }}$ is satisfied for model $A G_{\text {Network }}^{\prime}$, but it does not imply the satifaction of constraint $c_{1}$ (a forward-translated constraint, originally typed over $A T G_{\text {Components }}$ ), 


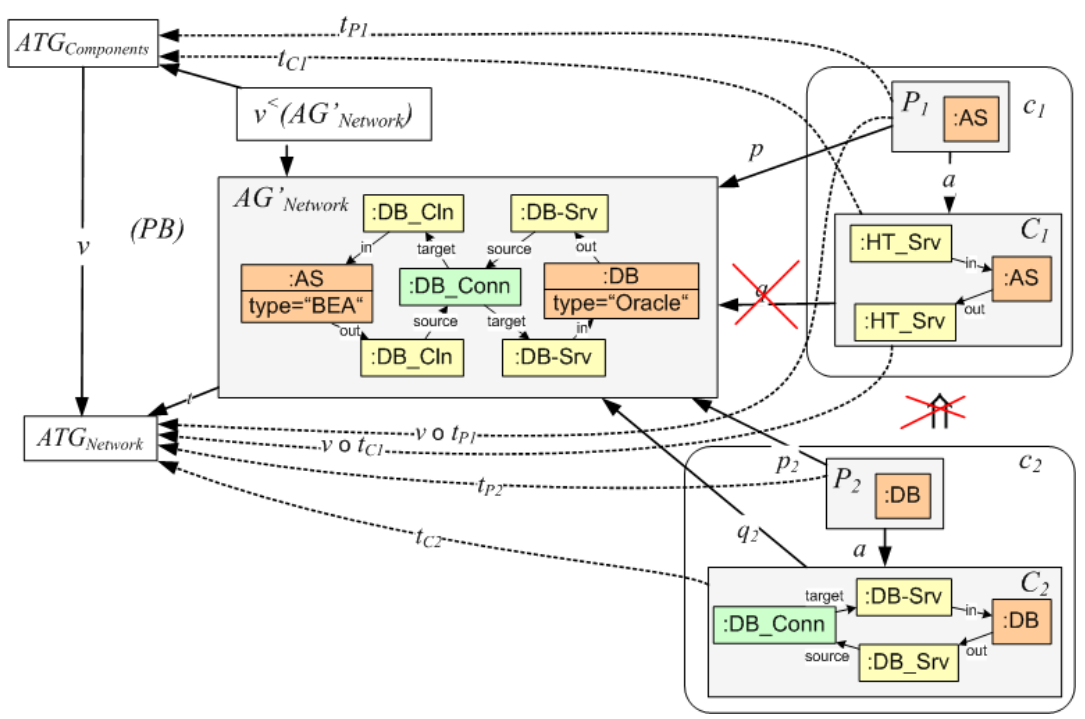

Fig. 9. Example 5.3: Constraint implication reflects backward-translated languages

since $c_{1}$ is not satisfied for model $A G_{\text {Network }}^{\prime}$. The view model $v^{<}\left(A G_{\text {Network }}^{\prime}\right)$ of model $A G_{N e t w o r k}^{\prime}$ over the view $A T G_{\text {Components }} \stackrel{v}{\longrightarrow} A T G_{\text {Network }}$ is obtained by constructing the pullback $(P B)$. The pullback object $v^{<}\left(A G_{N e t w o r k}^{\prime}\right)$ is typed over $A T G_{\text {Components }}$ and looks like $A G_{N e t w o r k}^{\prime}$ without the node :DB_Conn and its adjacent edges. Since the view model $v^{<}\left(A G_{N e t w o r k}^{\prime}\right)$ does not satisfy the original constraint $c_{1}$ typed over $A T G_{C o m p o n e n t s}$, it does not belong to the visual language defined by type graph $A T G_{C o m p o n e n t s}$ and a set of constraints $P C_{1}$ including $c_{1}$.

A view with constraints is consequently defined in Def. 5.4 as a view the (forward-translated) constraints of which are implied by the constraints of the original type graph. A VL is covered by views with constraints when its type graph is covered by the view type graphs, and additionally, its set of constraints consists of the union of the (forward translated) constraints of the views.

Definition 5.4 (View with constraints). Given attributed type graphs $A T G_{1}$ and $A T G_{2}$, constraints $P C_{1}$ and $P C_{2}$ over $A T G_{1}$ and $A T G_{2}$, respectively, and a view $\left(A T G_{1}, v_{1}\right)$ over $A T G_{2}$, then $\left(A T G_{1}, P C_{1}, v_{1}\right)$ is a view with constraints if $P C_{2} \Rightarrow v_{1}^{>}\left(P C_{1}\right)$.

$(A T G, P C)$ is covered by views with constraints $\left(A T G_{1}, P C_{1}, v_{1}\right)$ and $\left(A T G_{2}, P C_{2}, v_{2}\right)$ if $A T G$ is covered by $\left(A T G_{1}, v_{1}\right)$ and $\left(A T G_{2}, v_{2}\right)$, and $P C=v_{1}^{>}\left(P C_{1}\right) \cup v_{2}^{>}\left(P C_{2}\right)$.

Thm. 5.1 now extends Thm. 4.1 to views with constraints and states the condition for integration and decomposition of views with constraints.

Theorem 5.1 (Integration and decomposition with constraints). Let $(A T G, P C)$ be covered by the views $\left(A T G_{i}, P C_{i}, v_{i}\right)$ for $i=1,2$. If $\left(A G_{i}, t_{i}\right) \models P C_{i}$ are consistent models of $\left(A T G_{i}, v_{i}\right)$ via $\left(A G_{0}, t_{0}\right)$ then we have for the integration $(A G, t)$ that $A G \models P C$.

Vice versa, for the decomposition of $(A G, t)$ into view-models $\left(A G_{i}, t_{i}\right)$ with $i=1,2$ it holds that $A G_{i} \models$ $P C_{i}$.

Proof. Given the integration $(A G, t)$ we have that $v_{1}^{<}(A G, t)=\left(A G_{1}, t_{1}\right)=P C_{1}$. Now Fact 5.2 shows that this is equivalent to the fact that $A G \models v_{1}^{>}\left(P C_{1}\right)$. Analogously we have that $A G \models v_{2}^{>}\left(P C_{1}\right)$, and altogether $A G=P C$ because $P C=v_{1}^{>}\left(P C_{1}\right) \cup v_{2}^{>}\left(P C_{2}\right)$ by Def. 5.4.

Vice versa, $A G \models P C$ implies $A G \models v_{i}^{>}\left(P C_{i}\right)$ for $i=1,2$ and hence $A G_{i}=v_{i}^{<}(A G) \models P C_{i}$ by Fact 5.2 .

Example 5.4. The integration/decomposition diagram in Fig. 10 equals the diagram in Example 4.2 but is extended now by two constraints $c_{1}$ and $c_{2}$ which define views with constraints $\left(A T G_{\text {Components }}, P C_{C o m p}, v_{1}\right)$ and $\left(A T G_{\text {Connections }}, P C_{C o n n}, v_{2}\right)$. The consistent view models $G_{\text {Components }}$ and $G_{C o n n e c t i o n s}$, as shown in 


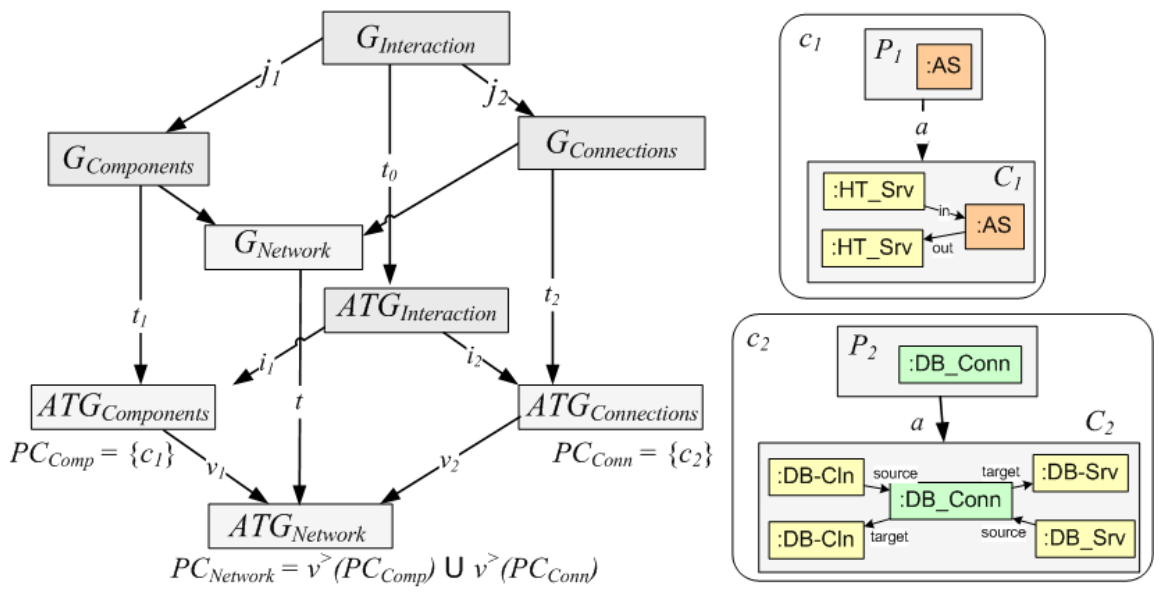

Fig. 10. Example 5.4: Integration and decomposition with constraints

Fig. 5, satisfy the respective constraints. It can be easily checked that the integration $G_{\text {Network }}$ (see Fig. 5) satisfies the forward-translated constraints $v_{1}^{>}\left(P C_{C o m p}\right)$ and $v_{2}^{>}\left(P C_{C o n n}\right)$. Vice versa, starting with model $G_{\text {Network }}$, we can decompose it into the two view-models $\left(G_{\text {Components }}, t_{1}\right)$ and $\left(G_{\text {Connections }}, t_{2}\right)$ such that each view-model satisfies the respective constraints.

\section{Related Work}

From a theoretical point of view, the concepts and results in this paper are closely related to the abstract framework of institutions, introduced by Goguen and Burstall [GB84] as general framework for data type specifications. An institution $I N S T=(\mathbf{S I G}, \operatorname{Mod}, S e n,=)$ consists of a category SIG of signatures, a contravariant functor $M o d: \mathbf{S I G}^{o p} \rightarrow$ CAT assigning to each signature $S I G$ a category $\operatorname{Mod}(\operatorname{SIG})$ of models, a functor $\operatorname{Sen}: \mathbf{S I G} \rightarrow$ Sets defining a set $\operatorname{Sen}(S I G)$ of sentences over $S I G$, and a satisfaction relation $=$, where $(M, \varphi) \in=$, written $M \models \varphi$, means that model $M$ satisfies sentence $\varphi$. The most prominent classical example is the institution $E Q S I G=(\mathbf{S I G}, A l g, E q n s, \models)$ of equational signatures, where SIG is the category of algebraic signatures, $A l g(S I G)$ the category of $S I G$-algebras and $S I G$-homomorphisms, Eqns $(S I G)$ the set of equations over $S I G$, and $A=e$ means that algebra $A$ satisfies equation $e$. In our paper, the concepts are defining an institution $A T G=($ ATGraphs, Mod, Constr,$\models)$ of attributed type graphs, where ATGraphs is the category GAGraphs restricted to attributed type graphs, $\operatorname{Mod}(A T G)$ is the category GAGraphs $_{\text {ATG }}$ of attributed graphs $A G$ typed over $A T G, C o n s t r(A T G)$ is the set of graph constraints of $A T G$-typed graphs, and $A G \models c$ means that attributed graph $A G$ satisfies constraint $c$. Fact 5.1 in our paper corresponds to the well-known satisfaction condition for institutions, and our main Theorem 5.1 means that the institution $A T G$ has amalgamation based on pushouts of attributed type graphs with constraints.

Viewpoint-oriented software development is well-known in the literature [GEMT00, GMT99, EEHT97], however identifying, expressing, and reasoning about meaningful relationships between view models is hard [NFK03]. Up to now existing formal techniques for visual modeling of views and distributed systems by graph transformation support the definition of non-hierarchical views which require a common fixed data signature [EEPT06, GDdL05]. This is in general not adequate for view-oriented modeling where only parts of the complete type graph and signature are known and necessary when modeling a view of the system. Moreover, hierarchical relations between views could not be defined on the typing and data type level resulting in a lack of composition and decomposition techniques for view integration, verification, and analysis.

In [AdLG07] domain specific languages are defined using graphical and textual views based on the metamodeling approach used in the $A T o M^{3}$ tool. In this approach the language designer starts with the common (integrated) meta-model and selects parts of the meta-model as different diagram views. So a common abstract meta-model is missing allowing to define hierarchical relations between the models.

In [RGH08] abstract graph views are defined, abstracting from specification details allowing a convenient usage of modules. To fulfill this purpose, reference relations have been introduced for the definition of 
mapping between view elements and abstract model elements (e.g. the database). Given this relations, there are different semantics for modifying view objects which are not studied yet in full detail. In comparison with the presented approach, generalized attributed graph morphisms have a unique formal semantics on the one hand and they provide the flexibility to define hierarchical relations on the other hand.

As a related approach xlinkit [NCEF07] provides rule-based link generation in web content management systems. In this approach semantics are defined using first order logic allowing automatic link generation to manage large document repositories. According to its purpose, this approach is limited to XML documents using XPath and XLink and thus requires an XML based storage format for models.

For related work concerning (nested) graph constraints we refer to [EEPT06, EEHP06, HP05].

Recently, the Query/View/Transformation Specification (QVT), Version 1.0 has been released by the OMG [QVT08]. Here, views are perceived as complex queries to select model parts. Despite its name, the main application area for QVT is model-to-model transformation. Queries and views are seen as special transformations. Transforming views at different meta-model levels, and ensuring consistency of views and view models for such transformations is not yet an issue of the QVT standard and tools.

QVT transformations are based on MOF meta-models and OCL [OCL03], a textual specification language providing constraint and object query expressions on meta-models that cannot be otherwise be expressed by diagrammatic notation. The combination of meta-models and OCL is closely related to our approach based on type graphs and graph constraints. In fact, the relationship has been discussed in our previous paper [WTEK06], where we identified a set of OCL constraints which can be translated to graph constraints. The combination of graph transformation rules for VL definition and graph constraints is as expressive as a meta-model with OCL constraints. This was shown e.g. in [BKPPT00], where a graph-based semantics for OCL is proposed by translating OCL constraints into expressions over graph rules. Vice versa, Cabot et al. present an approach to analyze graph transformation rules based on an intermediate OCL representation [CCGdL08]. Here, rules are translated to OCL with the purpose of verifying their correctness and allowing for interoperability with standards-based model-driven development tools.

\section{Conclusion}

In this paper we have studied the interaction and integration of views and the restriction of views along type hierarchies. The main result shows under which condition models of these views can be composed to a unique integrated model. The condition is called consistency of view models which means roughly that the models agree on the interaction type of the views. Vice versa, each model can be decomposed up to isomorphism into consistent models of given views. The paper is based on an extended version of typed attributed graph morphisms which allow changes of the type graph including those of data signatures and domains. In Thm. 1 we have considered visual languages based on meta-models given by attributed type graphs without constraints. In Thm. 2 we have shown that the main result can be extended to visual languages including constraints. Full proofs of all technical lemmas used in this paper and some extended results are given in our technical report [EEEP09].

An important consequence of our work is that we provide the ability to rapidly compose "small" visual languages both at the view (type graph) level and at the view-model level, thus laying the formal basis for multi-view modeling environments. Hence, rather than a "one modeling language does all" approach, we favor a confederation of small, relatively orthogonal visual languages for different system aspects. Future work is planned to investigate the interplay of views and models with behaviour, which is related to the field of merging behavioural models $\left[\mathrm{BCE}^{+} 06, \mathrm{UC} 04\right]$.

The concept of type hierarchies should allow a language designer to adapt language definitions by performing model transformations at an abstract hierarchy level and "inheriting" the transformation results at the more concrete levels of the hierarchy. Work is in progress to analyze model transformations for hierarchically structured visual languages.

Future work is planned to implement our formal approach by extending our graph transformation engine AGG [AGG09], a tool supporting visual modeling and analysis of typed, attributed graph transformation systems. Type graphs with inheritance model the underlying structure of the visual language used. The extension will offer means for structuring type graphs by hierarchies to enable language designers to compose / decompose visual languages at different abstraction levels. We will extend the underlying notion of typed attributed graph morphisms in AGG to the more general notion of $G A G$-morphisms and provide algorithms for checking the consistency conditions for integration and decomposition of view models. 


\section{References}

[AdLG07] F. P. Andrés, J. de Lara, and E. Guerra. Domain Specific Languages with Graphical and Textual Views. In A. Schürr, M. Nagl, and A. Zündorf, editors, Third International Symposium of Application of Graph Transformation with Industrial Relevance (AGTIVE'07), volume 5088 of LNCS, pages 79-94. Springer, 2007.

[AGG09] TFS-Group, TU Berlin. $A G G, 2009$. http://tfs.cs.tu-berlin.de/agg.

[Arb04] Farhad Arbab. Reo: A channel-based coordination model for component composition. Mathematical Structures in Computer Science, 14(3):329-366, June 2004.

[BBE ${ }^{+}$07] Benjamin Braatz, Christoph Brandt, Thomas Engel, Frank Hermann, and Hartmut Ehrig. An approach using formally well-founded domain languages for secure coarse-grained IT system modelling in a real-world banking scenario. In Proc. Australasian Conf. on Information Systems (ACIS'O'7), 2007.

$\left[\mathrm{BCE}^{+} 06\right]$ G. Brunet, M. Chechik, S. Easterbrook, S. Nejati, N. Niu, and M. Sabetzadeh. A Manifesto for Model Merging. In Proc. Workshop on Global Integrated Model Management(GaMMa'06), pages 4-12. ACM Press, 2006.

[BKPPT00] P. Bottoni, M. Koch, F. Parisi-Presicce, and G. Taentzer. Consistency Checking and Visualization of OCL Constraints. In UML 2000 - The Unified Modeling Language, volume 1939 of LNCS. Springer, 2000.

[CCGdL08] J. Cabot, R. Clarisó, E. Guerra, and J. de Lara. Analysing graph transformation rules through ocl. In Intern. Conf. on Theory and Practice of Model Transformations, volume 5063 of LNCS, pages 229-244. Springer, 2008.

[EEEP08] H. Ehrig, K. Ehrig, C. Ermel, and U. Prange. Consistent Integration of Models Based on Views of Visual Languages. In J.L. Fiadeiro and P. Inverardi, editors, Proc. Fundamental Approaches to Software Engineering (FASE'08), volume 4961 of $L N C S$, pages 62-76. Springer, 2008.

[EEEP09] H. Ehrig, K. Ehrig, C. Ermel, and U. Prange. Generalized Typed Attributed Graph Transformation Systems based on Morphisms Changing Type Graphs and Data Signatures. Technical Report TR 2009-08, Fak. IV, Technische Universität Berlin, 2009. http://www.eecs.tu-berlin.de/menue/forschung/forschungsberichte/2009.

[EEHP06] H. Ehrig, K. Ehrig, A. Habel, and K.-H. Pennemann. Theory of Constraints and Application Conditions: From Graphs to High-Level Structures . Fundamenta Informaticae, 74(1):135-166, 2006.

[EEHT97] G. Engels, H. Ehrig, R. Heckel, and G. Taentzer. A combined reference model- and view-based approach to system specification. Int. Journal of Software and Knowledge Engineering, 7(4):457-477, 1997.

[EEPT06] H. Ehrig, K. Ehrig, U. Prange, and G. Taentzer. Fundamentals of Algebraic Graph Transformation. EATCS Monographs in Theor. Comp. Science. Springer, 2006.

[EM85] H. Ehrig and B. Mahr. Fundamentals of Algebraic Specification 1: Equations and Initial Semantics, volume 6 of EATCS Monographs on Theoretical Computer Science. Springer, Berlin, 1985.

[GB84] J. A. Goguen and R. M. Burstall. Introducing institutions. In Proceedings of the Carnegie Mellon Workshop on Logic of Programs, pages 221-256. Springer-Verlag, 1984.

[GDdL05] E. Guerra, P. Diaz, and J. de Lara. A Formal Approach to the Generation of Visual Language Environments Supporting Multiple Views. In Proc. IEEE Symposium on Visual Languages and Human-Centric Computing (VL/HCC'05), IEEE Computer Society, Dallas, Texas, USA, September 2005.

[GEMT00] M. Goedicke, B. Enders, T. Meyer, and G. Taentzer. ViewPoint-Oriented Software Development: Tool Support for Integrating Multiple Perspectives by Distributed Graph Transformation. In Conf. on Tools and Algorithms for the Construction and Analysis of Systems, Berlin, Germany, volume 1785 of LNCS, pages $43-47$. Springer, 2000.

[GMT99] M. Goedicke, T. Meyer, and G. Taentzer. ViewPoint-oriented Software Development by Distributed Graph Transformation: Towards a Basis for Living with Inconsistencies. In Proc. 4th IEEE Int. Symposium on Requirements Engineering (RE'99), June 7-11, 1999, University of Limerick, Ireland. IEEE Computer Society, 1999.

[HP05] A. Habel and K.-H. Pennemann. Nested constraints and application conditions for high-level structures. In H.-J. Kreowski, U. Montanari, F. Orejas, G. Rozenberg, and G. Taentzer, editors, Formal Methods in Software and Systems Modeling, volume 3393 of Lecture Notes in Computer Science, pages 294-308. Springer, 2005.

[MOF06] Object Management Group. Meta-Object Facility (MOF), Version 2.0, 2006. http://www.omg.org/technology/ documents/formal/mof .htm.

[NCEF07] Ch. Nentwich, L. Capra, W. Emmerich, and A. Finkelstein. xlinkit: A Consistency Checking and Smart Link Generation Service. In Dept. of Computer Science, editor, University College London, 2007.

[NFK03] B. Nuseibeh, A. Finkelstein, and J. Kramer. Viewpoints: Meaningful relateionships are difficult. In Proc. Intern. Conf. on Software Engineering (ICSE). IEEE Computer Society, 2003.

[OCL03] Object Management Group. UML 2.0 OCL Specification, 2003. http://www.omg.org/docs/ptc/03-10-14.pdf.

[OMG07] Object Management Group. Unified Modeling Language: Superstructure - Version 2.1.1, 2007. formal/07-02-05, http://www. omg.org/technology/documents/formal/uml.htm.

[QVT08] Object Management Group. Meta Object Facility (MOF) 2.0 Query/View/Transformation Specification. Version 1.0 formal/08-04-03. http://www.omg.org/spec/QVT/1.0/, 2008.

[RGH08] U. Ranger, K. Gruber, and M. Holze. Defining Abstract Graph Views as Module Interfaces. In A. Schürr, M. Nagl, and A. Zündorf, editors, Third International Symposium of Application of Graph Transformation with Industrial Relevance (AGTIVE'07), LNCS, pages 117-133. Springer, 2008.

[UC04] S. Uchitel and M. Chechik. Merging Partial Behavioural Models. In Proc. 12th Int. ACM SIGSOFT Symposium on Foundations of Software Engineering, pages 43-52. ACM Press, 2004.

[WTEK06] J. Winkelmann, G. Taentzer, K. Ehrig, and J. Küster. Translation of Restricted OCL Constraints into Graph Constraints for Generating Meta Model Instances by Graph Grammars. In D. Varro and R. Bruni, editors, Proc. Graph Transformation and Visual Modeling Techniques (GT-VMT'06), ENTCS. Elsevier Science, 2006. 